\title{
Technology update: bronchoscopic thermal vapor ablation for managing severe emphysema
}

This article was published in the following Dove Press journal:

Medical Devices: Evidence and Research

30 September 2014

Number of times this article has been viewed

\author{
Daniela Gompelmann ${ }^{1,2}$ \\ Ralf Eberhardt ${ }^{1,2}$ \\ Felix JF Herth ${ }^{1,2}$ \\ 'Pneumology and Critical Care \\ Medicine, Thoraxklinik at University \\ of Heidelberg, ${ }^{2}$ German Center for \\ Lung Research, Heidelberg, Germany
}

\begin{abstract}
Bronchoscopic thermal vapor ablation (BTVA) is an endoscopic lung volume reduction therapy that presents an effective treatment approach in patients with severe upper lobe-predominant emphysema. By instillation of heated water vapor, an inflammatory reaction is induced, leading to fibrosis and scarring of the lung parenchyma, resulting in lobar volume reduction. Clinical single-arm trials demonstrated great outcomes, with significant improvement of lung function, exercise capacity, and quality of life. As the BTVA-induced local inflammatory response that seems to be essential for the desired lobar volume reduction can be associated with transient clinical worsening, strict monitoring of the patients is required. In future, the balance between efficacy and safety will constitute a major challenge. This review summarizes the BTVA procedure, the mechanism of action, and the results of the clinical trials, including the efficacy and safety data.
\end{abstract}

Keywords: emphysema, bronchoscopy, bronchoscopic thermal vapor ablation

\section{Introduction}

Chronic obstructive pulmonary disease (COPD) is a widespread disease associated with high mortality and morbidity. Chronic inflammatory processes, bronchoconstriction, airway remodeling, and emphysematous destruction of lung parenchyma lead to airflow limitation and hyperinflation, resulting in greater breathing effort. The main symptoms are dyspnea, low exercise capacity, and reduced quality of life. So far, there is no curative treatment approach, and thus the aim of medical care is directed at relieving of symptoms, improving quality of life, and slowing progression. Besides pharmacologic therapy, exercise training, oxygen therapy, and ventilatory support, lung volume reduction surgery (LVRS), developed in the 1950s by Brantigan et al, offers an effective symptomatic therapeutic approach. ${ }^{1}$ The aim of LVRS is to reduce the hyperinflation to optimize the respiratory mechanics, leading to improved lung function and physical performance. The surgical procedure, however, is associated with high morbidity and mortality. Therefore, LVRS was initially abandoned and first reintroduced in the $1990 \mathrm{~s}$ by Cooper et al. ${ }^{2}$ The most comprehensive clinical trial of LVRS was the randomized National Emphysema Treatment Trial (NETT), which compared 608 patients who underwent LVRS to 610 patients who received medical therapy. ${ }^{3}$ Following LVRS, patients experienced improvements of pulmonary function, exercise capacity, and health-related quality of life. However, the results of this trial also confirmed considerable mortality, particularly in patients with non-upper lobepredominant emphysema and high baseline exercise capacity, and thus stimulated the search for alternative approaches.
Correspondence: Daniela Gompelmann Pneumology and Critical Care Medicine, ThoraxKlinik at University of Heidelberg, 5 Amalienstrasse, Heidelberg 69126, Germany

Tel +4962213968087

Fax +496221396 I 202

Email daniela.gompelmann@med.uniheidelberg.de 
In the last decade, endoscopic lung volume reduction (ELVR), which basically imitates LVRS but with less attendant risk, was developed, and constitutes a new therapy for patients with severe emphysema. Several techniques of ELVR were developed in recent years, including valve therapy, coil implantation, bronchoscopic thermal vapor ablation (BTVA), polymeric lung volume reduction (PLVR), and the creation of airway bypasses. However, only the first three methods are currently performed. Due to a lack of investor funds, PLVR is (despite promising initial data) not available at present. The creation of extra-anatomic airway bypasses did not lead to sustainable benefit, so that this technique is no longer performed in emphysema patients.

While the endoscopic modalities differ in patient selection, mechanism of action, and side effects, the prerequisite of a successful use of all these techniques is the evidence of advanced emphysema associated with a forced expiratory flow in 1 second $\left(\mathrm{FEV}_{1}\right)<45 \%$ and a residual volume $(\mathrm{RV})$ of at least $>150 \%$, preferably $>200 \%$. The reversible valve therapy is the technique with the most comprehensive published data, and is already used extensively in Europe. This technique represents the blocking method that is only effective in patients with low interlobar collateral ventilation. Coil implantation and BTVA belong to the irreversible nonblocking methods that are the preferred techniques in patients with high collateral ventilation. Because the majority of patients have significant interlobar collateral ventilation and thus do not respond favorably to valve treatment, nonblocking devices, which are independent of collateral ventilation, present an alternative effective therapeutic approach. While coil implantation leads to lung volume reduction by placement of up to ten nitinol coils resulting in parenchymal compression, BTVA induces lobar volume reduction by inflammatory processes by instillation of heated water vapor.

\section{Bronchoscopic thermal vapor ablation (BTVA)}

BTVA (Uptake Medical Corporation, Seattle, WA, USA) was first described in 2009, and is the latest development of the ELVR techniques. By instillation of heated water vapor, a local inflammatory reaction is induced, leading to fibrosis and shrinkage, with the result of target lung volume reduction (TLVR). Therefore, it is currently the only technique where no implants are left. Furthermore, the segmental application of heated water vapor allows targeted therapy of emphysematous destroyed lung segments, and thus BTVA has the potential to manage intralobar heterogeneous emphysema. BTVA has not been approved, and so this technique is performed within clinical trials. A randomized controlled trial of BTVA is under way in Europe and Australia.

\section{Planning a bronchoscopic thermal vapor ablation treatment}

BTVA presents a therapeutic option for patients with heterogeneous upper lobe-predominant emphysema who are symptomatic despite medical therapy. The following selection criteria should be fulfilled: $\mathrm{FEV}_{1} 20 \%-45 \%$, RV $>150 \%$, diffusion capacity $\left(\mathrm{DL}_{\mathrm{CO}}\right)>20 \%$ and 6-minute-walk-distance $(6-\mathrm{MWD})>140 \mathrm{~m}$. The presence of severe emphysema and the heterogeneous distribution of emphysema must be confirmed by high-resolution computed tomography (HRCT) scan as $1 \mathrm{~mm}$ slices using a at least a 16-slice scanner. One exclusion criterion for BTVA is the presence of a bulla of more than a third of the lobar volume; however, apart from that, the severity of emphysematous destruction seems not to play a significant role in the efficacy of BTVA. Prior to the intervention, the airway target for procedure, as well as the vapor dose that depends on the density and volume of the targeted lung tissue, is identified on the basis of the patient's preinterventional HRCT by using dedicated software: the InterVapor Personalized Procedure Program (IP3) (Figure 1). For each patient, an IP3 data file with a 3-D reconstruction images of the patient's airways is created demonstrating the anatomical figures to simplify the procedure and specifying the treatment time and thus the vapor dose.

\section{Procedure of bronchoscopic thermal vapor ablation}

The BTVA system consists of two compartments ${ }^{4}$ :

- InterVapor catheter (Figure 2): this nonreusable InterVapor catheter is used to deliver the heated water vapor from the InterVapor generator to the targeted lung compartment; it is equipped with an inflatable balloon at the distal tip to occlude the airway during the treatment; the proximal end of the catheter is connected to the InterVapor generator.

- InterVapor generator (Figure 3): the InterVapor generator is an electronically controlled pressure vessel that generates the water vapor and delivers the calculated amount of vapor.

The catheter is introduced through a $2.8 \mathrm{~mm}$ working channel of a conventional flexible bronchoscope, and is positioned in the airway of the targeted lung region. Thereby, the 3-D reconstruction of the IP3 is used to determine the airway location for treatment. Afterwards, the balloon at the distal tip of the catheter is inflated to occlude the bronchus (Figure 4). Then, the $75^{\circ} \mathrm{C}$-heated water vapor is delivered within $3-10$ seconds 


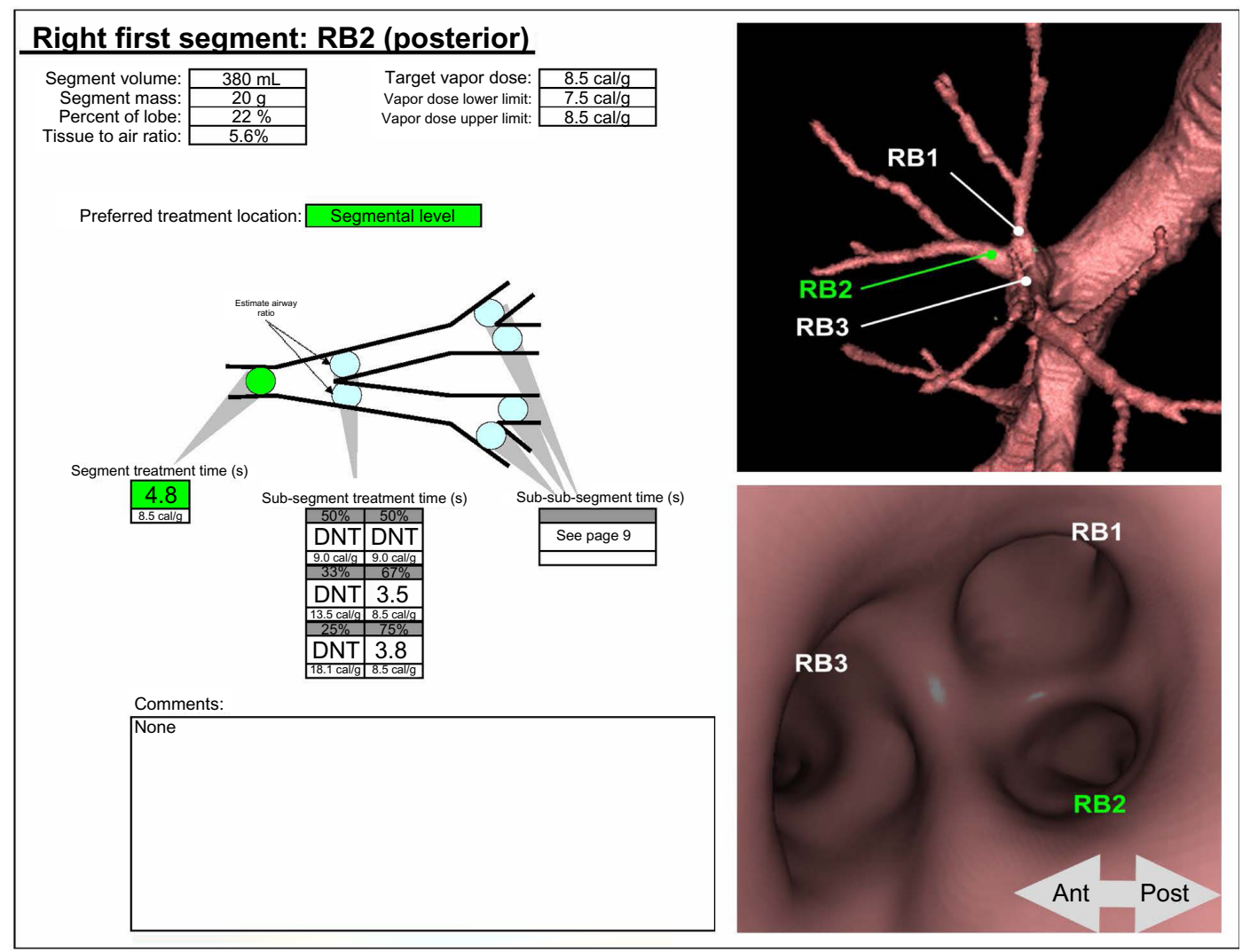

Figure I InterVapor Personalized Procedure Program (IP3).

Note: Courtesy of Uptake Medical Corporation, Seattle, WA, USA. In this example, the target for BTVA is the segment 2 of the right upper lobe. The preferred treatment location is on the segmental level. In case of BTVA of the total segment 2, the vapor dose is $8.5 \mathrm{cal} / \mathrm{g}$, the treatment time is 4.8 seconds. If the physician prefers to perform BTVA on the subsegmental level, the vapor dose and the treatment time change according to the estimated size of the subsegmental airways. Never apply InterVapor twice to the same region. Abbreviations: DNT, Do Not Treat; Ant, anterior; Post, posterior.

depending on the vapor dose $(7.5-8.5 \mathrm{cal} / \mathrm{g})$. Thereafter, the balloon is deflated and the catheter can be inserted into the next treatment site, such that the time between two vapor treatments should be 3 minutes or greater.

\section{Mechanism of action}

By instillation of heated water vapor, a localized inflammatory reaction is induced. The treated area heals with fibrosis,

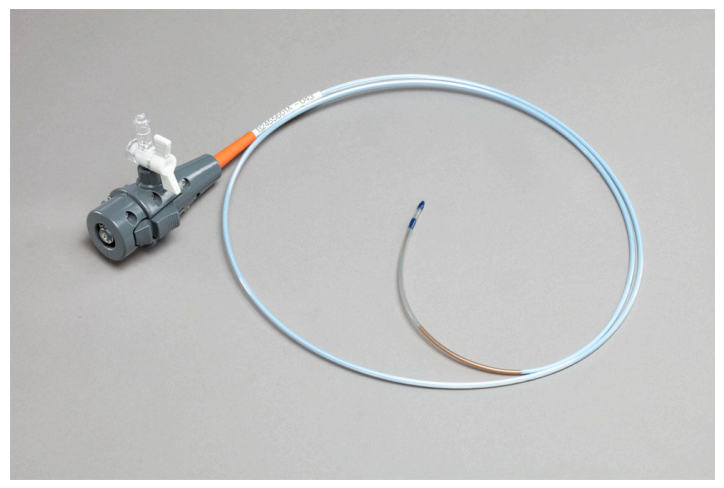

Figure 2 InterVapor catheter. Courtesy of Uptake Medical Corporation, Seattle, WA, USA. scarring, and shrinkage, thus resulting in lobar volume reduction. Heat-induced ablation of lung tissue was assessed in different animal trials. ${ }^{5,6}$ In a 2010 study, 26 dogs were treated by bronchoscopic steam injection. ${ }^{5}$ Three months following steam delivery, histological examination revealed alveolar damage (necrosis and fibrosis) and airway damage (bronchitis, bronchiolitis, and bronchopneumonia). In five dogs, histological examination revealed acute bronchopneumonia and microabscess formation, but staining for bacteria was negative. No coagulative necrosis was detected, suggesting that there is no high-temperature mechanism that is responsible for tissue ablation. Besides histological alteration, changes of lobar pulmonary blood flow was evaluated by intravenous injection of fluorescence-labeled microspheres prior to and following steam application. Immediately following steam injection, a significant reduction of lobar blood flow was observed. This effect was sustained over an observation period of 3 months. Therefore, it seems that the reduction of regional blood flow has impact on the physiological response to the BTVA procedure. It was hypothesized that mild heat insulted the ischemia and led to 


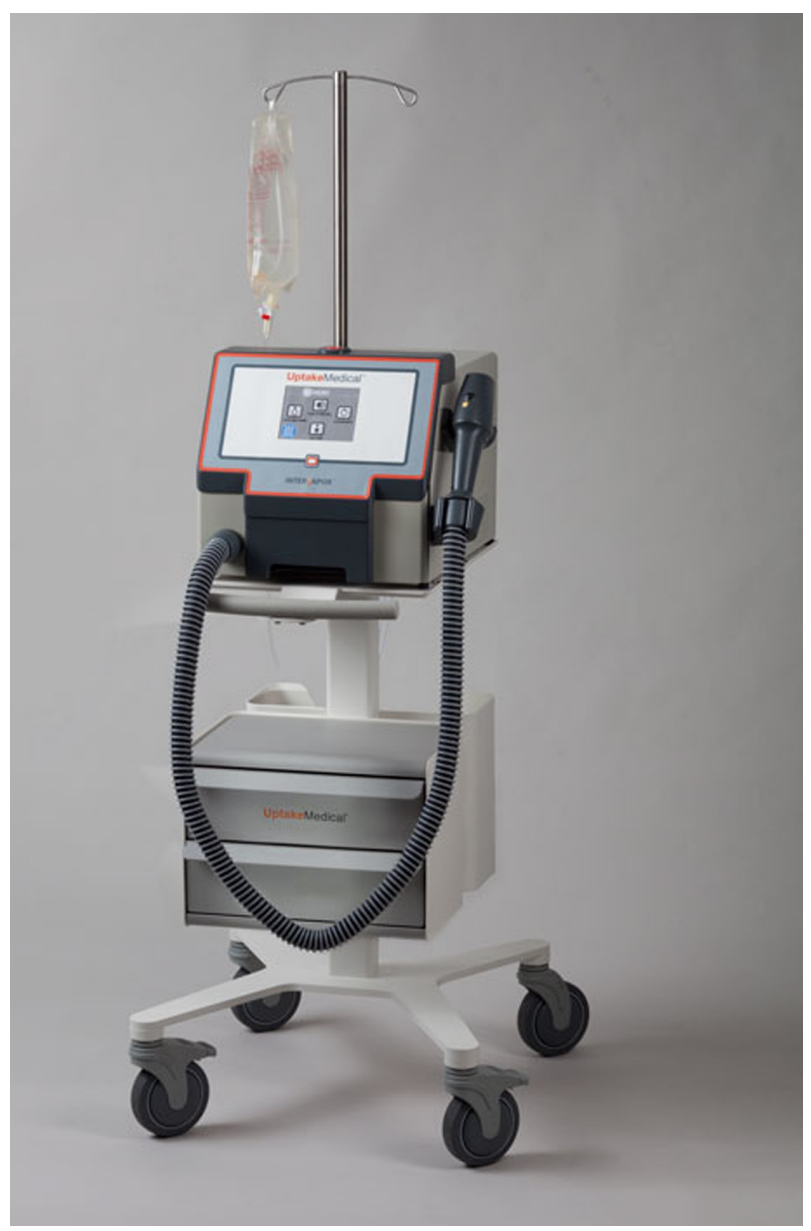

Figure 3 InterVapor generator. Courtesy of Uptake Medical Corporation, Seattle, WA, USA.

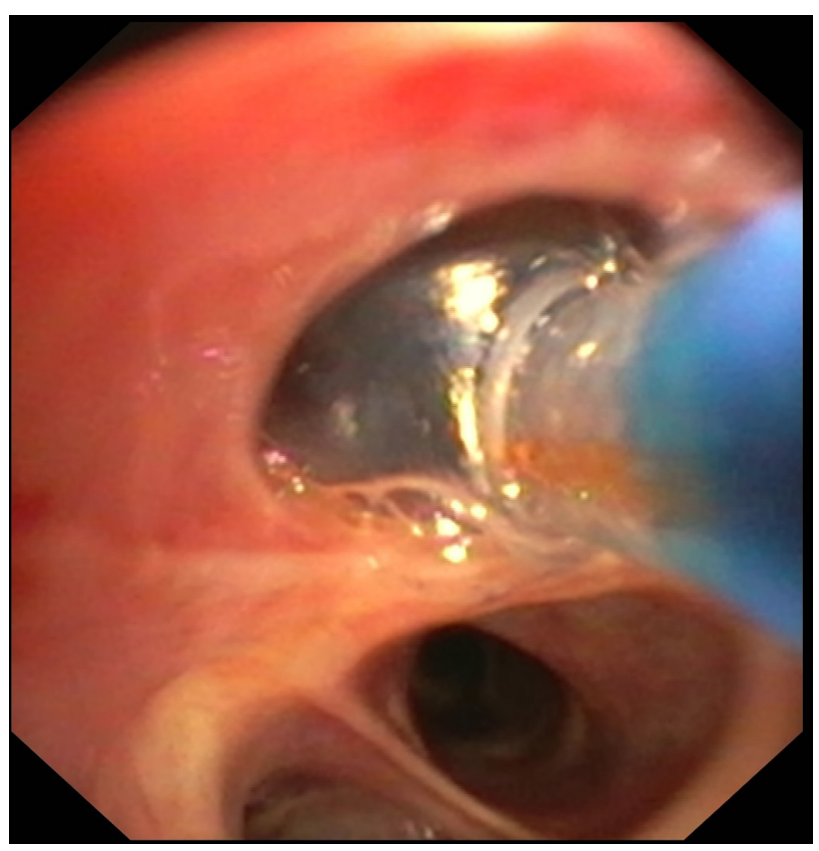

Figure 4 Endoscopic image.

Notes: The InterVapor catheter is introduced in the airway. The balloon at the distal tip of the catheter is inflated to occlude the airway. an apoptotic mechanism. In another trial, the effect of BTVA was examined in a canine model of emphysema. ${ }^{6}$ In the first few days following the BTVA procedure, inflammatory reactions, including cough, sputum, fever, and elevation of white blood cell counts, occurred. Ninety days following the procedure, histopathology revealed peribronchial, peribronchiolar, parenchymal, and alveolar septal fibrosis. However, only in a few slides chronic inflammation was observed. Therefore, it seems that an inflammatory reaction occurs immediately following the procedure, but is resolved within 3 months following BTVA. At this time point, mature fibrosis and remodeling are the predominant effects.

\section{Clinical profile, experience, and knowledge Safety and feasibility}

A pilot trial related to the feasibility and safety of BTVA in emphysema patients was published by Snell et al in $2009 .{ }^{7}$ In this trial, eleven patients with severe upper lobe-predominant emphysema with a mean $\mathrm{FEV}_{1}$ of $0.77 \mathrm{~L}$ (32\% predicted) and an RV of $4.16 \mathrm{~L}$ (219\% predicted) were treated by unilateral BTVA. Nine patients received BTVA treatment of the right upper lobe, two patients of the left upper lobe. In total, 33 segments of the upper lobes were treated. The mean vapor dose was $4.9 \mathrm{cal} / \mathrm{g}$. The mean treatment time was 4.6 seconds, the mean total procedure time was 22 minutes. The procedure was well tolerated in all patients. During follow-up, five serious adverse events, including COPD exacerbation, pneumonitis, and tachycardia, were noted. All serious adverse events required hospitalization, but resolved without sequelae. At 6 months following intervention, CT scans showed a mean lobar volume reduction of $16 \%$. The reduction of lobar volume was correlated with an improvement of health-related quality of life, as measured by the modified Medical Research Council (mMRC) dyspnea scale and St George's Respiratory Questionnaire (SGRQ). However, no notable change of lung-function parameters could be observed. In summary, the results of this pilot trial demonstrated the feasibility of BTVA with an acceptable safety profile, but only modest efficacy.

\section{Efficacy}

To enhance the effectiveness of BTVA, predictive factors for favorable outcome were sought. In a retrospective analysis, the heterogeneity index (HI) was identified as a predictor for excellent outcome following BTVA. ${ }^{8} \mathrm{HI}$ is defined as the tissue:air ratio of lower lobe:upper lobe, and was calculated using PW2 software (Vida Diagnostics, Iowa City, IA, USA). 
A subset of eight patients with more heterogeneous disease $\left(\mathrm{HI} \geq 1.2\right.$ ) had greater improvements in $\mathrm{FEV}_{1}$ and 6-MWT compared to all eleven patients, whereas SGRQ improved similarly in both groups.

To increase the efficacy of BTVA, not only patient selection was improved in a subsequent trial but also vapor dose was increased. This approach was justified by results of a preclinical animal study demonstrating a dose-dependent volume reduction. The first study, with a higher range of heat dose applied to humans, was a prospective multicenter single-arm trial conducted in Australia, the US, and Europe. ${ }^{9}$ Forty-four patients with upper lobe-predominant emphysema and an $\mathrm{HI}$ $\geq 1.2$ were enrolled. Mean $\mathrm{FEV}_{1}$ was $31.4 \% \pm 7.5 \%$, and mean RV was $237.0 \% \pm 50.1 \%$. All patients were treated unilaterally with BTVA using a vapor dose of $10 \mathrm{cal} / \mathrm{g}$. Twenty patients received BTVA in the left upper lobe, 24 patients in the right upper lobe. The mean procedure time was 29 minutes. There were no adverse events during bronchoscopic intervention. Within the first 6 months of treatment, 29 serious adverse events were observed, wherein COPD exacerbation, pneumonia, and respiratory tract infection were the most common serious complications. One patient died 67 days following BTVA, due to exacerbation of end-stage COPD. Regarding efficacy, the TLVR was $716 \pm 99 \mathrm{~mL}$, representing a $48 \%$ volume loss at 6 months. Furthermore, a significant improvement of lung-function parameters, including $\mathrm{FEV}_{1}(+141 \pm 62 \mathrm{~mL}$, $P<0.001)$, vital capacity $(+271 \pm 72 \mathrm{~mL}, P<0.001)$, and RV $(-406 \pm 113 \mathrm{~mL}, P<0.001)$ was documented. Exercise capacity measured by 6-MWT and health-related quality of life measured by the SGRQ and mMRC dyspnea scale also improved significantly at 6 months. Additional data from a 12-month follow-up revealed that lobar volume changes were stable. ${ }^{10}$ However, the magnitude of benefit related to the lung-function parameters, 6-MWD and health-related quality of life was less than that observed at 6 months, maybe indicating the progression of COPD or compensatory hyperinflation.

\section{Local inflammatory response}

During the first 4 weeks following intervention, laboratory tests revealed an increase of inflammatory markers (white blood cell count, C-reactive protein, neutrophils), and chest $\mathrm{X}$-ray frequently showed an opacification in the treated area, demonstrating that the effect of BTVA seems to be associated with an inflammatory response. ${ }^{9,11}$ This localized inflammatory response, which peaked within the first 2-4 weeks, was characterized by cough, sputum, increased dyspnea, fever, and mild hemoptysis. In the single-arm multicenter trial, 16 of 44 patients experienced respiratory adverse events requiring antibiotic therapy and/or glucocorticosteroids following BTVA. However, patients who developed a symptomatic inflammatory response had greater improvements in efficacy outcome than patients without respiratory adverse events. Significant differences were observed in lobar volume and $\mathrm{RV}$ over a 1 -year period and in $\mathrm{FEV}_{1} 3$ months following BTVA. Therefore, the localized inflammatory response seems to be essential for the desired TLVR. ${ }^{11}$

However, the inflammatory reaction often requires additional observation, medication, and hospitalization. Therefore, strong monitoring of patients following BTVA is crucial. A prophylactic broad-spectrum antibiotic for 14 days is recommended, and also glucocorticosteroids are advised to alleviate symptoms. For each patient, it is of great importance to balance the benefits with safety considerations. For example, patients with concomitant immune-system disorders or immunosuppressive medication should be excluded from BTVA, because it is likely that these patients are at increased risk for bacterial superinfection.

\section{Predictive factors}

As already mentioned, the local inflammatory reaction seems to be a predictive factor for beneficial outcome following BTVA. ${ }^{11}$ Nonetheless, it is still important to seek further predictive factors that would presumably lead to an enhanced favorable benefit-risk profile.

Therefore, the impact of treated lobar volume on efficacy was evaluated. ${ }^{11}$ Patients with treated lobar volume $>1,700 \mathrm{~mL}$ experienced a lobar volume reduction of $73 \%$ at 3 months following intervention compared to $40 \%$ in patients with low treated lobar volume. However, these patients with a high treated lobar volume appeared to be at higher risk of hospitalization in the first 30 days. This observation suggests that patients with higher volumes have great benefit form BTVA, but also require increased vigilance in monitoring or an alteration in the treatment approach.

On the basis of this multicenter trial, further different post hoc group analyses with regard to the GOLD (Global initiative for chronic Obstructive Lung Disease) classification and the HI were performed. ${ }^{10}$ When entering the trial, 22 patients had GOLD stage III and 22 patients had GOLD stage IV disease severity. While the improvements at 6 months were similar in GOLD stage III and IV disease, the improvements at 12 months were most consistent in GOLD stage IV patients, though the difference was not statistically significant. The authors hypothesized that compensatory hyperinflation potentially plays a more important role in GOLD stage III than in GOLD stage IV patients, resulting in 
difference in efficacy 1 year following intervention. Serious adverse events occurred in 9 GOLD stage III and in 14 GOLD stage IV patients, so the difference in efficacy outcome has to be balanced by safety concerns.

With regard to the $\mathrm{HI}, 22$ patients with an $\mathrm{HI}>1.6$ experienced greater improvement in lung-function parameters, exercise capacity, and health-related quality of life compared to the 22 patients with an $\mathrm{HI}<1.6 .{ }^{10} \mathrm{~A}$ significant betweengroup difference was detected for lobar volume reduction and for RV.

Furthermore, the impact of fissure integrity on efficacy following BTVA was evaluated. Several trials demonstrated that interlobar fissure completeness, which seems to be a surrogate for low interlobar collateral ventilation, is associated with beneficial outcome following valve therapy- the blocking technique of ELVR. ${ }^{12,13}$ Therefore, the objective of one retrospective analysis was to assess the influence of fissure integrity on outcome following BTVA as a nonblocking technique. ${ }^{14}$ Of the 44 patients treated with BTVA, $86 \%$ had incompleteness in the relevant interlobar fissures. The results revealed that there is no-to-minimal association between the fissure integrity and BTVA-induced lobar volume reduction and improvements in clinical outcomes. The authors hypothesized that this objective fact is probably due to the mechanism of action of BTVA, which does not rely on occlusion of large bronchi, but leads to fibrosis of lung parenchyma that is unlikely to reinflate.

\section{Current trials}

A multicenter, randomized trial evaluating safety and efficacy following segmental, bilateral BTVA in patients with severe emphysema is under way in Europe and Australia. In the STEP-UP (Sequential Segmental Treatment of Emphysema with Upper Lobe Predominance, NCT01719263) study, patients with predominantly upperlobe emphysema, $\mathrm{HI}>1.2, \mathrm{FEV}_{1} 20 \%-45 \%$, RV $>150 \%$ and 6-MWD $>140 \mathrm{~m}$ are randomized to BTVA treatment or standard medical care. The BTVA is sequentially performed in one to two segments in the upper lobes of each lung. The idea of this sequential therapeutic approach is to enhance the safety aspects without compromising the efficacy. The results of this trial are expected in 2015/2016.

\section{Conclusion}

BTVA is an effective treatment approach in patients with upper lobe-predominant emphysema. By inducing an inflammatory reaction, BTVA leads to fibrosis and scarring of the lung parenchyma. As it is independent of interlobar collateral ventilation, BTVA is available in a great number of patients. Significant improvement of clinical outcome parameters following BTVA was observed in clinical trials. However, data are limited, and so far there are no results of a prospective randomized controlled trial. The results of the first clinical randomized STEP-UP trial are expected in $2015 / 2016$. One main focus will rely on evaluation of further predictive factors for good outcome. As the effect of BTVA is based on an irreversible inflammatory reaction, patient selection is crucial. The balance between efficacy and safety will constitute a major challenge.

\section{Disclosure}

The authors report no conflicts of interest in this work.

\section{References}

1. Brantigan OC, Mueller E, Kress MB. A surgical approach to pulmonary emphysema. Am Rev Respir Dis. 1959;80(1 Part 2):194-206.

2. Cooper JD, Patterson GA, Sundaresan RS, et al. Results of 150 consecutive bilateral lung volume reduction procedures in patients with severe emphysema. J Thorac Cardiovasc Surg. 1996;112(5):1319-1329.

3. Fishman A, Martinez F, Naunheim K, et al. A randomized trial comparing lung-volume-reduction surgery with medical therapy for severe emphysema. N Engl J Med. 2003;348(21):2059-2073.

4. Uptake Medical. Corp InterVapor [system operator's manual]. Tustin (CA): Uptake Medical; 2013.

5. Emery MJ, Eveland RL, Eveland K, Couetil LL, Hildebrandt J, Swenson ER. Lung volume reduction by bronchoscopic administration of steam. Am J Respir Crit Care Med. 2010;182(10):1282-1291.

6. Tuck SA, Lopes-Berkas V, Beam S, Anderson JC. Bronchoscopic thermal vapor ablation in a canine model of emphysema. Int $J$ Chron Obstruct Pulmon Dis. 2012;7:21-31.

7. Snell GI, Hopkins P, Westall G, Holsworth L, Carle A, Williams TJ. A feasibility and safety study of bronchoscopic thermal vapor ablation: a novel emphysema therapy. Ann Thorac Surg. 2009;88(6):1993-1998.

8. Herth FJ, Eberhardt R, Ernst A, et al. The efficacy of bronchoscopic thermal VAPOR ablation (BTVA) in patients with upper lobe emphysema: the impact of heterogeneity of disease. Poster presented at: American Thoracic Society 2010 International Conference; May 14-19, 2010; New Orleans, LA.

9. Snell G, Herth FJ, Hopkins P, et al. Bronchoscopic thermal vapor ablation therapy in the management of heterogeneous emphysema. Eur Respir J. 2012;39(6):1326-1333.

10. Herth FJ, Ernst A, Baker KM, et al. Characterization of outcomes 1 year after endoscopic thermal vapor ablation for patients with heterogeneous emphysema. Int J Chron Obstruct Pulmon Dis. 2012;7:397-405.

11. Gompelmann D, Eberhardt R, Ernst A, et al. The localized inflammatory response to bronchoscopic thermal vapor ablation. Respiration. 2013;86(4):324-331.

12. Sciurba FC, Ernst A, Herth FJ, et al. A randomized study of endobronchial valves for advanced emphysema. $N$ Engl J Med. 2010;363(13): $1233-1244$.

13. Herth FJ, Noppen M, Valipor A, et al. Efficacy predictors of lung volume reduction with Zephyr valves in a European cohort. Eur Respir J. 2012;39(6):1334-1342.

14. Gompelmann D, Heussel CP, Eberhardt R, et al. Efficacy of bronchoscopic thermal vapor ablation and lobar fissure completeness in patients with heterogeneous emphysema. Respiration. 2012;83(5):400-406. 
Medical Devices: Evidence and Research

Dovepress

\section{Publish your work in this journal}

Medical Devices: Evidence and Research is an international, peerreviewed, open access journal that focuses on the evidence, technology, research, and expert opinion supporting the use and application of medical devices in the diagnosis, treatment and management of clinical conditions and physiological processes. The identification of novel devices and optimal use of existing devices which will lead to improved clinical outcomes and more effective patient management and safety is a key feature. The manuscript management system is completely online and includes a quick and fair peer-review system. Visit http://www. dovepress.com/testimonials.php to read real quotes from authors.

Submit your manuscript here: http://www.dovepress.com/medical-devices-evidence-and-research-journal 